\title{
NATURALISTIC STUDIES OF DRIVER DISTRACTION: EFFECTS OF ANALYSIS METHODS ON ODDS RATIOS AND POPULATION ATTRIBUTABLE RISK
}

\author{
Richard A. Young \\ Wayne State University School of Medicine \\ Detroit, Michigan, USA \\ Email: ryoun@med.wayne.edu
}

\begin{abstract}
Summary: When analyzing naturalistic driver performance data, different analysis methods can have large impacts on safety estimates for the condition being assessed. To illustrate, this paper reanalyzed the data for a secondary task (conversation on a hand-held cell phone) from the recently-released Virginia Tech Transportation Institute (VTTI) 100-Car databases, using a standard method for epidemiological analysis. It found substantially lower estimates for the odds ratio (OR), population exposure percent $\left(P_{e} \%\right)$, and population attributable risk percent (PAR\%) than with the VTTI analysis method. The crash/near-crash OR was reported by VTTI as 1.29, but was found to be 0.78 with the standard method, a reversal in direction from a potentially crash-increasing to a potentially crash-reducing effect. The $P_{e} \%$ for crashes/near-crashes was $12.5 \%$ using the VTTI method, but declined to $6.7 \%$ with the standard method. The PAR\% was reported as $3.6 \%$ but a population preventive fraction of $1.5 \%$ (a protective effect) was estimated by the standard method. The OR difference was traced to an "assumption bias" in the VTTI method that had unequal effects for the unexposed vs. exposed cases. The $P_{e} \%$ and PAR\% differences were traced to an error in the VTTI calculation of $P_{e} \%$. This bias and error were systemic in the VTTI analysis methods, overestimating OR, $P_{e} \%$, and PAR\% for all tasks examined. Future research should seek to better understand the epidemiologic analysis methods that are most appropriate in the new and emerging field of naturalistic driving research.
\end{abstract}

\section{INTRODUCTION}

Naturalistic driving studies (NDS) that use video cameras, GPS, lane tracking, radar to surrounding vehicles, and data acquisition systems installed in light ${ }^{1}$ and heavy ${ }^{2}$ vehicles to capture each moment of driving for weeks, months, or years per driver, have led to a paradigm shift in traffic safety research, particularly with regard to driver "inattention." One focus has been on mishaps recorded during the NDS, which generate estimates of the odds of a crash or near-crash (here termed an event) associated with various conditions, including a secondary task, defined as a "task, unrelated to driving, which requires subjects to divert attention resources from the driving task.”(1, p. xvi)

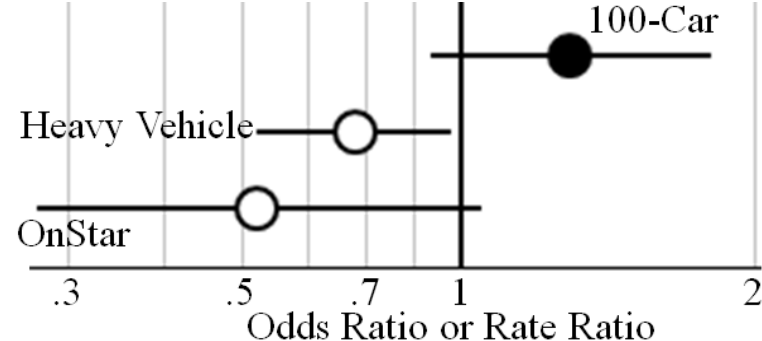

Figure 1. Three studies of cellular conversation
The VTTI “100-Car” NDS conducted from 2001 to 2004 collected data on driver behavior, including while performing secondary tasks in light vehicles. ${ }^{1}$ From these data, VTTI estimated the odds ratio (OR) for events associated with hand-held cell phone conversation (hereafter called Talk) to be 1.29 with a $95 \%$ confidence interval (CI) mostly above 1 (Fig. 1). ${ }^{(1, \text { Table 2.5) }}$ (An OR above 1 means the odds are higher than baseline driving.) VTTI estimated the OR for Talk as 0.78 (Fig. 1), with a CI below 1 , in another NDS, using heavy vehicles in fleets with no cell phone policy. ${ }^{(2, \text { Table } 29)}$ In a 
cohort study of 3 million vehicles equipped with the OnStar device, ${ }^{3}$ the rate ratio for airbagdeployment crashes during hands-free personal calls was 0.62 (Fig. 1), with a CI mostly below 1. (The OR and rate ratio are the same size if events are rare, such as airbag deployment crashes, which occur in OnStar-equipped vehicles at 8.18 per 100 million minutes of driving. ${ }^{3}$ ) It is clear by inspection of Fig. 1 that the $100-C a r$ Study ${ }^{1}$ Talk OR is in the opposite direction from other studies. ${ }^{2,3}$

While it is possible that this opposite result is due to random variation, or to demographic and/or device differences among the studies, the analysis methods employed should also be examined to determine whether they may have caused or contributed to the opposite result. For example, two other real-world studies estimated a risk ratio (RR) for Talk near 4, but these studies had a substantial positive bias from part-time driving in control periods ${ }^{4}$ and so need not be further considered. The current study explores the potential impact of different analysis approaches on the 100-Car OR estimates and related measures. Specifically, this paper conducts an independent analysis of recentlyreleased 100-Car Study databases, comparing a Standard Method to the VTTI Method of analysis. Although Talk is the only detailed example given, the OR results for other tasks are summarized.

\section{METHODS}

Epidemiological Notation and Terminology. Table 1 provides a notation for two analysis methods for estimating OR, $P_{e}$, and PAR\% in a case-control study. ${ }^{(5, \text { p. } 128 \& \text { p. } 137)}$ For ease of reference, this paper refers to these as the "Standard Method" (Table 1A), and the "VTTI Method" (Table 1B).

Table 1. Epidemiological notation. A. Standard Method. B. VTTI Method

\begin{tabular}{|c|c|c|c|c|c|c|c|c|c|c|c|}
\hline \multicolumn{6}{|c|}{ A. Standard Method } & \multicolumn{6}{|c|}{ B. VTTI Method } \\
\hline \multirow{4}{*}{ 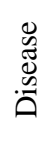 } & \multirow{4}{*}{$\begin{array}{l}\text { Yes } \\
\text { No }\end{array}$} & Exposed $X \dagger$ & Not Exposed $X \dagger$ & Total & $P_{e}$ & \multirow{4}{*}{$\underset{\mid>1}{\overrightarrow{0}}$} & \multirow{4}{*}{$\begin{array}{l}\text { Yes } \\
\text { No }\end{array}$} & Exposed $X \dagger$ & None $\neq$ & \multirow{4}{*}{$\begin{array}{c}\text { Total } \\
a+b \\
c+d\end{array}$} & $P_{e}$ \\
\hline & & $A$ & $B$ & $A+B$ & \multirow{3}{*}{$C /(C+D)$} & & & $a$ & $b$ & & \\
\hline & & $C$ & $D$ & $C+D$ & & & & $C$ & $d$ & & $c /(c+d)$ \\
\hline & & \multicolumn{3}{|c|}{$\mathrm{OR}=A D / B C$} & & & & \multicolumn{2}{|c|}{$\mathrm{OR}=a d / b c$} & & \\
\hline
\end{tabular}

$\dagger X=$ Supposed cause of disease $\quad \ddagger$ No exposure to any supposed cause of event Standard Method. Exposed $X$ connotes those who have been exposed to $X$, a "supposed cause of a disease."(5, p. 66) Not Exposed $X$ connotes those who have not been exposed to $X$. Those in the study group identified as having the disease under investigation ("Disease Yes”) are called cases. (5, p. 21) Those without the disease ("Disease No") are called controls. ${ }^{\text {(5, p. 41) }}$ The exposure-odds ratio (OR) is the ratio of the odds in favor of exposure among cases $(A / B)$ to the odds in favor of exposure among controls $(C / D)$. The exposure $O R$ is $(A / B) /(C / D)$, which algebraically reduces to $A D / B C$. The reference for those exposed to $X$ (i.e., $A$ and $C$ ), is those not exposed to $X$ (i.e., $B$ and $D$ ). The population exposure $\left(P_{e}\right)$ is the proportion of exposed in the population. ${ }^{(5, \text { p. 137) }} P_{e}$ may be estimated by the number of exposed controls divided by the total controls, or $C /(C+D),{ }^{(5, p .37 ; 6)}$ which, multiplied by 100 , forms $P_{e} \% . P A R \%$ is the incidence of disease in a population that is associated

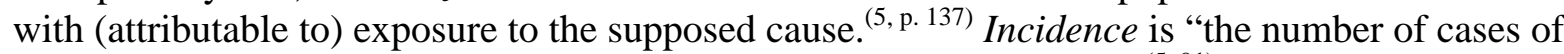
illness commencing...during a given period in a specified population." ${ }^{(5,91)}$ PAR\% gives the percentage of disease cases in the population that would be eliminated if the cause were eliminated. Eq. 1 gives PAR\% in terms of $P_{e}$ and risk ratio (RR). ${ }^{(5, \text { p. 137; 6) }}$

$$
\text { Population Attributable Risk } \%=\mathrm{PAR} \%=100 \times P_{e}(\mathrm{RR}-1) /\left(1+P_{e}(\mathrm{RR}-1)\right)
$$

With RR $<1$, the Population Preventive Fraction \% (here termed PPF\%) is used, defined as the

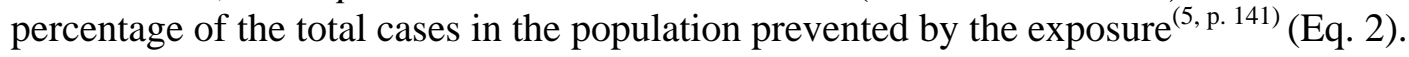

$$
\text { Population Preventive Fraction } \%=\mathrm{PPF} \%=100 \times P_{e}(1-\mathrm{RR})
$$

When the disease is uncommon in all compared exposure groups, the OR approximates the $\mathrm{RR},{ }^{6}$ and the OR may be substituted for $\mathrm{RR}^{6,7}$ in Eqs. 1 and 2, provided there is no confounding. ${ }^{8}$ Ref. 7 gives 
equations for estimating the $\mathrm{CI}$ for $\mathrm{OR}$ and $\mathrm{PAR} \%$. The $\mathrm{CI}$ gives the range over which the true value of the estimate may be found with a given probability (e.g. 95\%). Use of the CI for statistical hypothesis testing is not recommended in epidemiology: "Because statistical hypothesis testing promotes so much misinterpretation, we recommend avoiding its use in epidemiologic presentations and research reports." (9, p. 163) However, careful attention should still "be paid to the confidence interval, especially its width and its endpoints (the confidence limits),"(9, p. 163) but solely to assist estimation, not to test hypotheses. ${ }^{(9, \text { Chap. } 10)}$ The Standard Method is used for epidemiologic analyses wherein each exposure is independent (i.e., no interaction or effect modification between exposures). (In this paper, the general term exposure is used to refer to secondary tasks, rather than the term "risk factor," because case-control studies estimate odds and not risks.)

100-Car Databases. An Event database contained video clips of driver behavior that were 6 sec long, with 5 secs before and 1 sec after a "precipitating event," just before a crash or near-crash event. ${ }^{(1, \text { xii- }}$ ${ }^{\mathrm{xv})}$ A Baseline database contained 6-sec video clips of uneventful driving (and so lacked a precipitating event, crash, or near-crash). VTTI adapted epidemiologic methods to its NDS analyses by treating crashes and near-crashes (hereafter called events) as the equivalent of a "disease." In epidemiology, the 2x2 matrix entries such as in Table 1A are usually counts of subjects for the various conditions. VTTI adapted this method to NDS data by defining each 2x2 matrix entry as a count of video clips rather than subjects. Exposed $X$ then means exposed to a supposed cause (e.g., a secondary task) observed in a video clip. The VTTI report then stated (correctly) that its method met was a case-control design, ${ }^{(1, \text { p. viii) }}$ with multiple baseline clips (controls) sampled for each event (cases). (VTTI did not claim the design was "matched case-control" or "case-crossover" because Baseline and Event clips were only partially matched for vehicles and drivers, and not matched at all for GPS location and time of day.) However, the specific definitions of the individual cells in the 2x2 matrices in Table 1 differ substantially between the Standard and VTTI Methods.

Current Analysis Using Standard Method. The publicly-released databases containing the 100-Car Event and Baseline data were downloaded ${ }^{10}$ and imported to Excel 2010 for initial checks and Stata/IC 12.1 for Windows for final tabulations and analysis. There were 828 event rows and 19,616 baseline rows, which differed only slightly from the 830 events and 19,827 baseline "epochs" reported in the 100-Car Study. ${ }^{1}$ Fifteen rows in the Event database and 1 in the Baseline database had "No analyzed data” or "no video available," and were dropped, leaving 813 event clips and 19,615 baseline clips with usable data. The current study then adapted the Standard Method in Table 1A for NDS analysis. For example, Talk in the Event and Baseline databases was tabulated according to the following specific definitions of the counts for $A, B, C$, and $D$ in Table 1 A using Talk as an example: $A=$ Event clips where Talk was present;

$B=$ Event clips where Talk was not present ("No Talk");

$C=$ Baseline clips where Talk was present;

$D=$ Baseline clips where Talk was not present (“No Talk”).

VTTI Method. VTTI used its own unique method (Table 1B) instead of the Standard Method (Table 1A). The differences become clear after considering VTTI's unique definitions of $a$ - $d$. These were not accurately described in the original100-Car report, ${ }^{(1, \text { p. } 27)}$ but they were in a later VTTI report, ${ }^{11}$ reproduced below using Talk as an example:

$a$ = Event clips with Talk where the 100-Car driver "was at least partially at fault" or "where distraction played a role in the crash regardless of fault;",(11, p. 66)

$b=$ Event clips with no secondary task ("No Task") where the 100-Car driver was at fault;

$c=$ Baseline clips where Talk was present (same as $C$ in the Standard Method);

$d=$ Baseline clips with no secondary task ("No Task"). 
To calculate the exposure odds for secondary tasks, VTTI used $b$ and $d$ as a common reference, which they labeled "No Distraction," 11 but which is here labeled more neutrally as "No Task."

Concerns with VTTI Method. First, the VTTI Method defines $a$ more inclusively than $b$, because $a$ counts events "where distraction played a role in the crash," but $b$ does not. This permits confounding by assumption bias ${ }^{(5, \text { p. } 15)}$ - the video reductionists may have assumed that Talk was a distraction that "played a role in the crash" whenever Talk was observed in an Event clip, and hence counted all such clips in $a$. However, $b$ is restricted to at-fault events only, causing possible upward bias in the OR estimate. In contrast, the Standard Method as applied here avoids this bias by counting $A$ and $B$ events regardless of fault. Second, the VTTI Method erred in estimating $P_{e}$ from the definitions $a-d$. Note that $d$ is always smaller than $D$, because $d$ includes no baseline secondary tasks, whereas $D$ includes all such tasks except Talk. Hence, $c+d$ is always less than the true total number of baseline clips $C+D$. Using $c /(c+d)$ to estimate $P_{e}$, as per the VTTI Method in Table 1B, overestimates $P_{e}$. The error in $P_{e}$ is carried forward to overestimate PAR\% via Eq. 1 , and PPF\% via Eq. 2. The Standard Method simply divides $C$ by the known total baseline count $C+D$ to estimate $P_{e}$, and hence estimates of $P_{e}, \mathrm{PAR} \%$ and PPF\% are likely more consistent with true population values.

\section{RESULTS}

There was a substantial impact on the resultant OR and other metrics from the differences in the two Methods in their respective definitions of $b$ and $d$ vs. $B$ and $D$. The newly-calculated Talk data and results using the Standard Method are in Table 2A, and the Talk data and results used VTTI Method are in Table 2B, reproduced verbatim from Ref. 11 (its Table 6).

Table 2. Analysis of 100-Car Talk task. A. Standard Method B. VTTI Method

\begin{tabular}{|c|c|c|c|c|c|c|c|c|c|c|}
\hline \multirow{4}{*}{$\begin{array}{l}\text { Crash/Near- } \\
\text { Crash }\end{array}$} & \multicolumn{5}{|c|}{ A. Standard Method } & \multicolumn{5}{|c|}{ B. VTTI Method $^{(11, \text { Table 6) }}$} \\
\hline & \multirow{3}{*}{$\begin{array}{l}\text { Yes } \\
\text { No }\end{array}$} & Talk & No Talk & \multirow{4}{*}{$\begin{array}{c}\text { Total } \\
813 \\
19,615\end{array}$} & \multirow{4}{*}{$\begin{array}{l}P_{e} \% \\
6.8 \%\end{array}$} & \multirow{3}{*}{$\begin{array}{l}\text { Yes } \\
\text { No }\end{array}$} & Talk & No Task & \multirow{4}{*}{$\begin{array}{c}\text { Total } \\
281 \\
10,358\end{array}$} & \multirow[t]{2}{*}{$P_{e} \%$} \\
\hline & & 44 & 769 & & & & 44 & 237 & & \\
\hline & & 1,339 & 18,276 & & & & 1,299 & 9,059 & & $12.5 \%$ \\
\hline OR & $\begin{array}{l}\text { \% CI) } \\
\text { PPF\% }\end{array}$ & $0.78(0.56$ to 1.06$)$ & $\begin{array}{l}\text { o } 1.06) \\
\text { \% to } 3.2 \%)\end{array}$ & & & $\begin{array}{r}\text { OR }(95 \% \mathrm{CI}) \\
\text { PAR\% }\end{array}$ & $\begin{array}{l}1.29(0 \\
3.6 \%(\end{array}$ & $\begin{array}{l}\text { to } 1.80) \\
\text { o to } 4.1 \%)\end{array}$ & & \\
\hline
\end{tabular}

Root Cause of OR Difference. The Standard Method OR estimate is 0.78, which is in the opposite direction, with little overlap in the CI range, from the VTTI Method OR estimate of 1.29. The cause of this discrepancy cannot be: (1) the databases, which were essentially the same for both Methods; or (2) the Talk condition, because $a=A(44)$ and $c(1,299)$ is only $3 \%$ different than $C(1,339)$. The first possible cause is that $b$ (237) is smaller than $B$ (769). A partial reason for this is that all secondary tasks were discarded from $b$, but were retained in $\mathrm{B}$, except for Talk. However, this difference does not bias the OR, because similar treatment was given to $d$ and $D$ respectively. The determining cause for the OR difference is that $b$ counts only at-fault cases, while $B$ counts all cases, regardless of fault. This creates a bias in the VTTI Method which confounds its OR estimate, because the unexposed clips $b$ (entering the OR denominator) were restricted in terms of subject driver fault, but the exposed clips $a$ (entering the OR numerator) were not, because of the "assumption bias" described above. This is proven empirically because $a=A$, so the VTTI Method must have counted all events with Talk in $a$, regardless of fault. This was further verified by cross-tabulating the Talk events by the fault variable, resulting in 25 at-fault, 9 not-at-fault, and 10 unknown fault cases in $a$. A similar cross-tabulation for $b$ verified that the $237 b$ cases in Table $1 \mathrm{~B}$ were at-fault only, unlike $a$. This unequal treatment of $a$ and $b$ by the VTTI Method is the root cause of the OR difference between the Methods. The Standard Method avoids this bias because all event clips were treated equally in $A$ (Talk) and $B$ (No Talk), by counting all events regardless of driver fault. 
Root Cause of $P_{e}$ and PAR\% Differences. The Standard Method reduced the VTTI $P_{e} \%$ of $12.5 \%$ to $6.8 \%$, and reversed the VTTI PAR\% of $3.6 \%$ to a PPF\% of $1.5 \%$. That is, if Talk never occurred while driving, the VTTI Method predicts that events would decrease by 3.6\%, while the Standard Method predicts they would increase by $1.5 \%$, an opposite effect. As predicted in the "Concerns" section above, the $P_{e}$ and PAR\% differences are caused by the small $d$ of 9,059 in the VTTI Method, vs. the $D$ of 18,276 in the Standard Method. Hence, the total baseline count of 10,358 in Table 2B is far less than the true total baseline count of 19,615 in Table 2A, causing the denominator of $P_{e} \%$ to be too small in the VTTI Method, so $P_{e} \%$ is (falsely) elevated. The fact that the OR is less than 1 in the Standard Method then causes the PAR\% in the VTTI Method to reverse to a protective PPF\%.

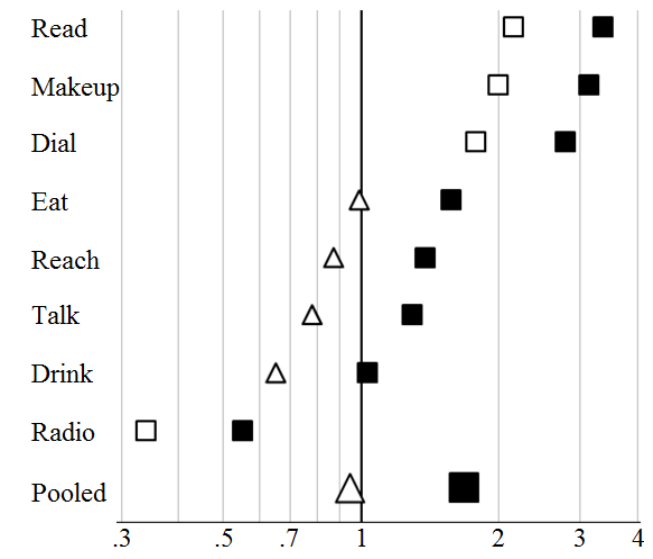

Figure 2. Task ORs. Solid symbols: VTTI Method. Open symbols: Standard Method
Systemic Bias and Error. The bias and error identified in the VTTI Method from the Talk analysis were systemic, affecting every published OR and PAR\% value in the 100 Car reports. ${ }^{1,11}$ Fig. 2 compares the OR estimates from the two methods for some representative secondary tasks. The VTTI Method (solid symbols) always produced higher OR estimates than the Standard Method (open symbols). Four of the 8 tasks (Eat, Reach, Talk, and Drink) and the pooled OR estimate reversed direction from above 1 for the VTTI Method to below 1 for the Standard Method (triangles).

"Hybrid Method" as Control. The Standard Method uses a "No Talk" reference for the unexposed condition rather than a common reference. Therefore, all secondary tasks

other than Talk are present in the $B$ and $D$ cells (and likewise for the $A$ and $C$ cells). A possible error that might arise is hinted at in a different context when analyzing multiple disease exposures that are simultaneously present: "When several exposures are being evaluated, nonexposure should correspond to the absence of all exposures. A common error...is to compare each exposed group with the complementary set of nonexposed, eschewing a single well-defined reference group. Doing so may result in causes appearing to be preventives and will generally lead to underestimates of effects."(12, p. 59) The above quote, although in an entirely different context, nonetheless raises concern that the Standard Method, which uses a complementary set of nonexposed, may underestimate the OR in a multi-exposure setting when it only analyzes a single exposure at a time. If the combined effect of all other tasks increases the odds of an event, then $B$ may be biased high relative to $D$, underestimating the OR. As a control, a Hybrid Method was developed that uses a common reference group as does the VTTI Method, but matches the fault conditions in $a$ and $b$ as does the Standard Method, thereby avoid the "assumption bias." (This Hybrid Method is a non-standard epidemiological design and is not recommended for general use; it is used here only as a control.) Table 3A shows the Hybrid Method result for at-fault cases, and Table 3B for all cases, regardless of fault.

Table 3. Hybrid Method as control. A. At-fault cases only. B. All cases, regardless of fault

\begin{tabular}{|c|c|c|c|c|c|c|c|c|c|c|}
\hline \multirow{5}{*}{$\begin{array}{l}\text { Crash/Near- } \\
\text { Crash }\end{array}$} & \multicolumn{5}{|c|}{ A. Subject Driver At-Fault } & \multicolumn{5}{|c|}{ B. All Cases, Regardless of Fault } \\
\hline & & Talk & No Task & \multirow{4}{*}{$\begin{array}{c}\text { Total } \\
271 \\
10,644\end{array}$} & $P_{e} \%$ & \multirow{3}{*}{$\begin{array}{l}\text { Yes } \\
\text { No }\end{array}$} & Talk & No Task & \multirow{4}{*}{$\begin{array}{c}\text { Total } \\
538 \\
10,644\end{array}$} & \multirow{2}{*}{$P_{e} \%$} \\
\hline & \multirow{2}{*}{$\begin{array}{l}\text { Yes } \\
\text { No }\end{array}$} & 25 & 246 & & & & 44 & 494 & & \\
\hline & & 1,339 & 9,305 & & N/A & & 1,339 & 9,305 & & N/A \\
\hline & $\begin{array}{r}\text { OR (95\% CI) } \\
\text { PPF\% }\end{array}$ & \multicolumn{2}{|c|}{0.71 (0.45 to 1.07$)$} & & & $\begin{array}{r}\text { OR (95\% CI) } \\
\text { PPF\% }\end{array}$ & \multicolumn{2}{|c|}{$0.62(0.44$ to 0.85$)$} & & \\
\hline
\end{tabular}

The results in Table 3 shows that using the common "No Distraction" reference in the Hybrid control lowers OR estimates to 0.71 and 0.62, vs. the original Standard Method OR of 0.78 in Table 2A. This control demonstrates empirically that the Standard Method does not underestimate the OR when 
evaluating a specific secondary task from an NDS dataset containing multiple tasks. $\left(P_{e} \%\right.$ and PPF\% metrics are shown as N/A or "Not Available" in Table 3 because the Standard Method equations do not correctly estimate those metrics when using a common reference, as noted earlier.)

\section{DISCUSSION AND CONCLUSION}

Differences between the VTTI 100-Car OR estimate for Talk ${ }^{1}$ and those from independent real-world studies $^{2,3}$ led to the question of whether methodological issues could underlie the differences. A Standard Method of epidemiological analysis using the 100-Car databases was compared to published results from the VTTI Method. The OR, $P_{e} \%$, and PAR\% estimates from the VTTI Method were consistently higher than those from the Standard Method for all secondary tasks tested. A bias and error in the VTTI Method were traced to a unique treatment of the "No Distraction" reference condition. ${ }^{1,11}$ The Standard Method was free of this bias and error, and estimated OR and $P_{e} \%$ effect sizes for Talk which were consistent with those in independent studies. ${ }^{2,3,13,14}$

Root Cause of Positive OR Bias. The VTTI Method ${ }^{1,11}$ caused high OR estimates because it counted only at-fault unexposed cases (which enter the OR denominator), but counted all cases, regardless of fault, for the exposed cases (which enter the OR numerator). This assumption bias is the logical fallacy of "begging the question," because the 100-Car investigators assumed secondary tasks were a "distraction that plays a role in the crash" during the video reduction, before the OR analysis was done, and then used the OR results to confirm that assumption. Hence, the VTTI Method positively biased the Talk OR estimate such that it reversed direction and became causal rather than preventive, compared to the Standard Method. The results from two independent studies of heavy vehicles ${ }^{2}$ and OnStar-equipped light vehicles ${ }^{3}$ were consistent with the Standard Method OR estimate, but not with the VTTI Method OR estimate.

Root Cause of Overestimated $P_{e}$ and PAR\%. The VTTI Method made an error in using the unexposed baseline count $d$ to estimate $P_{e}$ via the equation $c /(c+d)$. This error is empirically confirmed by the fact that the Talk $P_{e} \%$ of $12.5 \%$ as estimated by the VTTI Method is inconsistent with the population prevalence for Talk observed in two independent studies: (1) the percentage of $6.7 \%$ for Talk in the complete (not sampled) dataset of all Talk exposure from key-on to key-off in a vehicle for 108 subjects for week 1 of a 6 -week study in $2009-2010 ;{ }^{13}$ (2) the $6.7 \%$ upper end of the range of percentages of driving time in the U.S. occupied by hand-held cell phone conversation from direct observations of drivers stopped at intersections during daylight hours, in studies conducted every year from 2004-2010. ${ }^{14}$ By contrast, the $P_{e} \%$ for Talk estimated by the Standard Method (6.8\%) is consistent with these independent study results. ${ }^{13,14}$ With the VTTI Method, the overestimated $P_{e} \%$ then arithmetically overestimated PAR\%.

A control study found, after removing the "assumption bias," that the Talk OR estimate was robust to changing "No Talk" to a common reference of "No Task." The Talk OR estimates and their CIs remained below or near 1, proving empirically that the Standard Method does not underestimate the OR in an NDS setting. That is, including other secondary tasks in the unexposed condition makes little difference in estimating an individual task OR in a multi-task NDS. One explanation is that the pooled effect of all tasks other than Talk is about equal on the exposed and unexposed groups; i.e., the pooled task OR is near 1 (see Fig. 2), suggesting a balance between preventives and causes in the full 100-Car task set. The Standard Method is hence preferable to the VTTI or Hybrid Methods, because it estimates $\mathrm{OR}, P_{e} \%$, PAR\%, and PPF\% using simple formulas that give reasonably valid estimates consistent with independent studies. ${ }^{2,3,13,14}$ To obtain more precise estimates in future work will likely require the use of a common reference group with all exposures and their interactions simultaneously included in the analysis, requiring more advanced epidemiologic methods. ${ }^{15,16,17,18}$ 
In conclusion, future research should seek to better understand the epidemiologic analysis methods that are most appropriate in the new and emerging field of naturalistic driving research.

\section{REFERENCES}

${ }^{1}$ Klauer, S., Dingus, T., Neale, V., Sudweeks, J. \& Ramsey, D. (2006). The impact of driver inattention on near-crash/crash risk: An analysis using the 100-Car naturalistic driving study data. Washington, DC: NHTSA.

${ }^{2}$ Hickman, J., Hanowski, R. \& Bocanegra, J. (2010). Distraction in commercial trucks and buses: Assessing prevalence and risk in conjunction with crashes and near-crashes. Wash., D.C.: U.S. DOT, FMVCSA.

${ }^{3}$ Young, R. A. \& Schreiner, C. (2009). Real-world personal conversations using a hands-free embedded wireless device while driving: Effect on airbag-deployment crash rates. Risk Analysis, 29(2), 187-204.

${ }^{4}$ Young, R. A. (2012a). Cell phone use and crash risk: Evidence for positive bias. Epidemiology, 23(1), 116-118.

${ }^{5}$ Last, J. (2001). A Dictionary of Epidemiology (Fourth Ed.). NY: Oxford University Press.

${ }^{6}$ Cole, P. \& MacMahon, B. (1971). Attributable risk percent in case-control studies. Journal of Epidemiology and Community Health, 25(4), 242-244.

${ }^{7}$ Sahai, H. \& Khurshid, A. (1995). Statistics in Epidemiology: Methods, Techniques, and Applications Boca Raton, FL: CRC Press.

${ }^{8}$ Greenland, S. (1984). Bias in methods for deriving standardized morbidity ratio and attributable fraction estimates. Statistics in Medicine, 3(2), 131-141.

${ }^{9}$ Rothman, K., Greenland, S. \& Lash, T. (2008). Modern Epidemiology (3rd Ed.). PA: Lippincott.

10 100-Car databases at http://forums.vtti.vt.edu/index.php?/files/category/3-100-car-data/.

${ }^{11}$ Hankey, J. (2007). Comparison of odds ratios and population attributable risk for cell phone dialing and talking/listening on a handheld cell phone. VTTI, Blacksburg, VA. pp. 60-72 of brief filed to docket NHTSA-2007-28442. http://www.regulations.gov/\#!documentDetail;D=NHTSA2007-28442-0002.

${ }^{12}$ Rothman, K. (1986). Modern Epidemiology (1st Ed.). Boston: Little, Brown and Company.

${ }^{13}$ Funkhouser, D. \& Sayer, J. (2012). A naturalistic cell phone use census. TRB 2012 Annual Meeting, Paper 12-4104; January 22-26; Washington, DC. http://amonline.trb.org/1soumj/1.

${ }^{14}$ NHTSA. (2011). Driver electronic device use in 2010. Traffic Safety Facts: Research Note (DOT HS 811 517). Retrieved from http://www.distraction.gov/download/researchpdf/8052_TSF_RN_DriverElectronicDeviceUse_1206111_v4_tag.pdf.

${ }^{15}$ Greenland, S. \& Drescher, K. (1993). Maximum likelihood estimation of the attributable fraction from logistic models. Biometrics, 49(3), 865-872.

${ }^{16}$ Benichou, J. (2007). Biostatistics and epidemiology: measuring the risk attributable to an environmental or genetic factor. Comptes Rendus Biologies, 330(4), 281-298.

${ }^{17}$ Walter, S. (1980). Prevention for multifactorial diseases. American Journal of Epidemiology, 112(3), 409-416.

${ }^{18}$ Morgenstern, H. \& Bursic, E. (1982). A method for using epidemiologic data to estimate the potential impact of an intervention on the health status of a target population. Journal of Community Health, 7(4), 292-309. 